\title{
Familial ventricular tachycardia: a report of four families
}

\author{
Christopher Wren, Edward Rowland, John Burn, Ronald W F Campbell
}

\begin{abstract}
Four cases of familial ventricular tachycardia are presented. In each family the proband was an adolescent girl. Twelve members in the four families were affected and all were female. The clinical and electrocardiographic features were relatively constant within each family but there were striking differences between families. These differences argue against a common electrophysiological or pathophysiological basis for the ventricular tachycardia.
\end{abstract}

Familial ventricular tachycardia is rare. Most reported cases have the long QT syndrome or are associated with familial cardiomyopathies. We report four families in which ventricular tachycardia occurred in at least two members. There were important differences between families but the clinical and electrocardiographic features were strikingly consistent within each family.

\section{Case reports}

FAMILY A

The proband (fig 1), an apparently healthy 14 year old girl, was found to have an irregular pulse at a routine medical examination. Physical examination, including hearing, was otherwise normal. The resting electrocardiogram showed sinus bradycardia, a PR interval of $120 \mathrm{~ms}$, a QT interval of $420 \mathrm{~ms}$ (QTc $438 \mathrm{~ms}$ ), and very prominent $U$ waves, particularly in right precordial leads (fig 2d). During the recording there were several salvos of non-sustained multiform ventricular tachycardia. She was then symptom free but within 12 months she had several episodes of dizziness and presyncope, after which a 24 hour electrocardiographic recording showed frequent ventricular extrasystoles $(>1800$ per hour) and up to 80 episodes of multiform ventricular tachycardia per hour (at rates up to 160 per minute) (fig 3), with almost complete suppression of arrhythmia overnight. She was symptom free during this recording. The arrhythmia was unaltered during an exercise stress test. A Valsalva manoeuvre produced a flat heart rate response with no electrocardiographic changes and no ventricular arrhythmia.

The patient's mother who for several years had been taking phenytoin for "epilepsy" was also investigated. Her syncope always occurred during activity but not necessarily on exertion. Her electrocardiogram was remarkably similar to that of her daughter (fig 2c) and 24 hour electrocardiographic recording showed up to 1800 ventricular extrasystoles per hour and up to 155 episodes of ventricular tachycardia per hour (rate up to 190 per minute) (fig 4) with almost complete suppression overnight.

The proband's father's electrocardiogram was normal. That of her younger sister also showed prominent $U$ waves (fig $2 e$ ) but 24 hour electrocardiographic recording showed no arrhythmia. Electrocardiograms from the patient's maternal grandmother (fig $2 b$ ) and great-grandmother (fig 2a) also show abnormal $\mathrm{U}$ waves. Other family members have normal electrocardiograms (fig 1).

Despite treatment with $\beta$ blockers, her syncope recurred and a high left cervical sympathectomy was performed. Postoperatively she continued treatment with a $\beta$ blocker but had further syncope. Prolonged electrocardiographic monitoring showed persisting complex ventricular ectopic activity and no ventricular tachycardia, but there was profound sinus and nodal bradycardia. The $\beta$ blocker was stopped, and since then she has been symptom free, with no recurrence of tachycardia or bradycardia.

The mother is symptom free on treatment with a $\beta$ blocker although ventricular arrhythmias are still present on ambulatory electrocardiographic recordings.

\section{FAMILY B}

The proband, a 13 year old symptom free girl, was found to have an irregular pulse during a viral illness. Physical examination was otherwise normal. A resting electrocardiogram showed ventricular bigeminy with $T$ wave inversion in inferolateral leads. Ambulatory monitoring showed frequent ventricular extrasystoles ( $>2250$ per hour), with prolonged periods of bigeminy. Only rarely were there two consecutive sinus beats, but when this did occur a large abnormal $U$ wave was present. Ventricular tachycardia was frequent (1-24 episodes an hour) and was bidirectional (fig 5) or multiform. The tachycardia rate was 170 beats per minute. During a Valsalva manoeuvre the ventricular arrhythmias were abolished but the recording quality precluded measurement of the QT interval. On exercise stress testing the number of ventricular extrasystoles initially increased and then fell, with abolition by stage 5 of the Bruce protocol. The extrasystoles returned during recovery. A cross sectional echocardiogram was normal.

Within nine months severe episodes of dizziness developed but she was not syncopal. On 


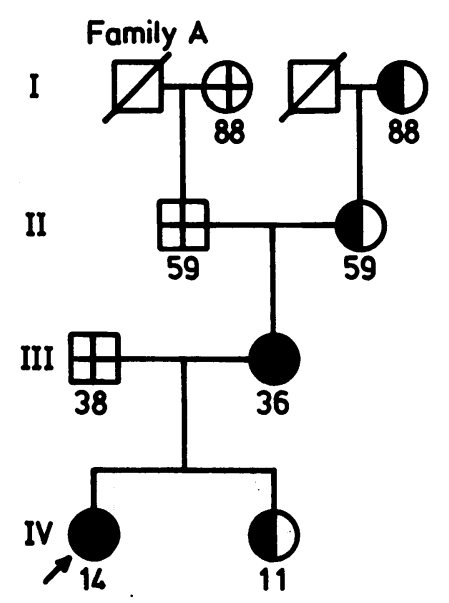

\section{Family B}

\section{I}

II $\bigoplus_{43}$

III

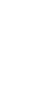

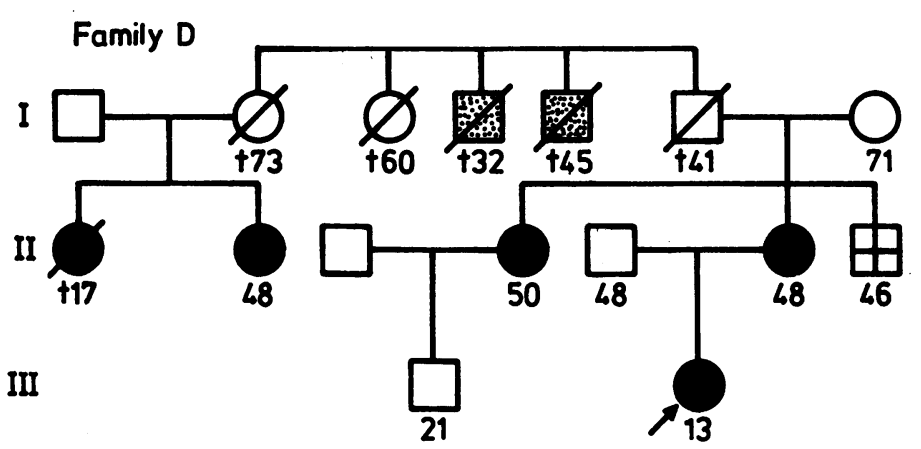

Figure 1 Pedigrees of the four families. propranolol both her symptoms and the ventricular tachycardia persisted. Mexiletine and atenolol were not tolerated. On flecainide she continued to have almost incessant ventricular bigeminy and short runs of ventricular tachycardia and her symptoms were controlled for a few months but then returned. At an electrophysiology study rapid, non-sustained, multiform ventricular tachycardia could be induced by premature stimuli but induction was not reproducible and could not be used for drug testing. Monophasic action potential recordings showed no evidence of dispersion of ventricular repolarisation. Subsequent treatment with disopyramide produced no improvement in either her symptoms or the arrhythmia. Currently her symptoms are well controlled by amiodarone although monitoring shows that the arrhythmia persists.

The patient's mother volunteered that she had also had an irregular pulse for many years, with no history of syncope or dizziness. Her physical examination was normal. Her electrocardiogram showed frequent ventricular arrhythmias and 24 hour recording showed frequent ventricular extrasystoles $(>2800$ per hour) and many episodes ( $>18$ an hour) of non-sustained bidirectional or multiform ventricular tachycardia (fig 6). Her arrhythmias were also suppressed during the Valsalva manoeuvre and on peak exercise. Electrocardiograms from the patient's brother, sister, and father, and her mother's brother, sister, and parents were normal (fig 1 ). (a)

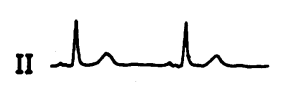
(b)<smiles>CCCCC(C)CCC(C)C</smiles><smiles>[SiH3]</smiles>

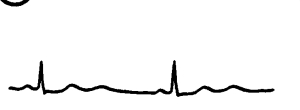<smiles>[131I-]</smiles>

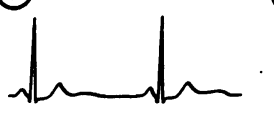<smiles>CCC(C)C</smiles><smiles>[R2]C(C)CCC(C)CCC</smiles><smiles>CCCC(C)CCC(C)C</smiles>

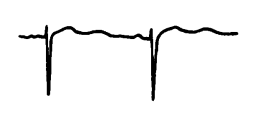<smiles>CCCC(C)CCCC(C)C</smiles><smiles>CCC(C)(C)CC</smiles>
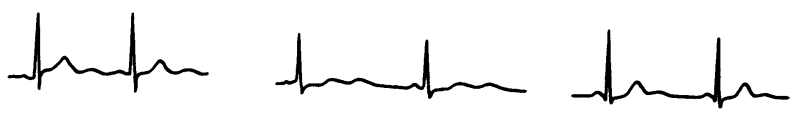

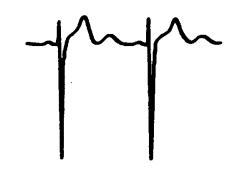

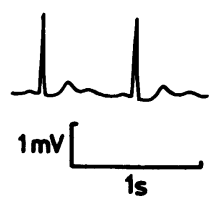

Figure 2 Electrocardiograms (leads II, V2, V5) from members of family $A$ showing prominent $U$ waves. a, maternal great-grandmother (I.4); b, maternal grandmother (II.2); $c$, mother (III.2); d, proband (IV.1); e, younger sister (IV.2). 


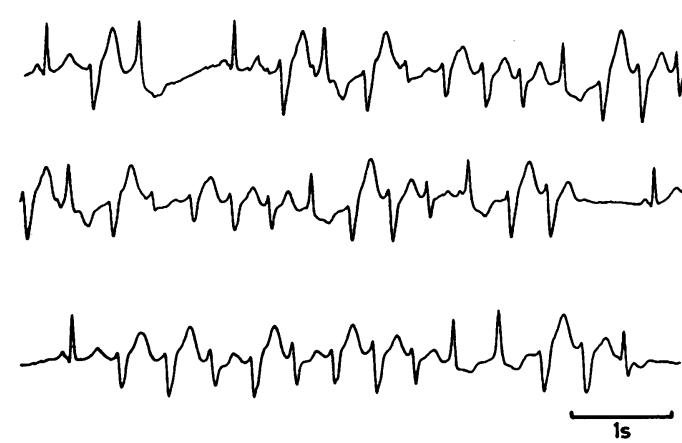

Figure 3 Multiform ventricular tachycardia recorded during ambulatory electrocardiographic monitoring in the proband from family $A$.

\section{FAMILY C}

The proband, a 15 year old girl, was referred after three episodes of presyncope on exercise. Physical examination was normal. The resting electrocardiogram showed ventricular bigeminy and non-sustained ventricular tachycardia. The QT interval could not be measured because consecutive sinus beats were not recorded. Ambulatory electrocardiographic recording confirmed frequent multiform ventricular tachycardia at 160 beats per minute (fig 7). Echocardiography showed a slightly dilated left ventricle. The ejection fraction by radionuclide angiocardiography was normal. Treatment with amiodarone provoked thyrotoxicosis and did not relieve her symptoms. While being treated with flecainide, and with a right atrial AAI pacemaker, she has been symptom free for two years.

In 1966 the patient's mother had heart failure, atrial flutter, and multiform ventricular tachycardia a few months after the birth of her second child (the proband). Cardiac catheterisation and angiography led to a diagnosis of "congestive cardiomyopathy". Although the arrhythmias persisted, the heart failure resolved and there were no symptoms for the next 14 years. In 1980, after a syncopal attack, the electrocardiogram showed frequent ventricular arrhythmias including non-sustained ventricular tachycardia. There was no clinical evidence of heart failure and the left ventricle was only slightly dilated on $M$ mode echocardiography. The mother died suddenly one year later, presumptively from a ventricular arrhythmia.

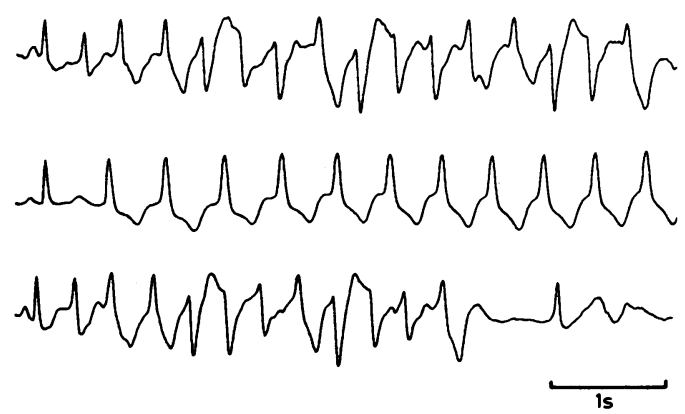

Figure 4 Ventricular tachycardia recorded during ambulatory electrocardiographic monitoring of the mother of the proband of family $A$. The ventricular tachycardia is at times multiform and irregular and at other times stable and monomorphic.

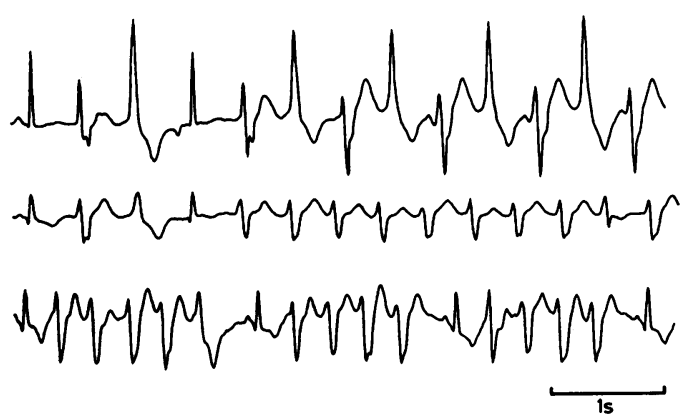

Figure 5 Ventricular tachycardia recorded during ambulatory electrocardiographic monitoring of the proband from family $B$. Several types of ventricular tachycardia were seen including bidirectional tachycardia (upper trace), regular monomorphic tachycardia (middle trace), and irregular multiform ventricular tachycardia

(lower trace).

At necropsy the left ventricle was mildly dilated but there was no important macroscopic or histological abnormality.

The patient's older sister had also presented to another hospital with ventricular arrhythmias and cardiac enlargement and died suddenly at the age of 13 years.

\section{FAMILY D}

The proband developed palpitation at the age of 10 years. Investigations at that time were normal; no arrhythmia was seen. She presented again three years later with palpitation, chest pain, and dyspnoea on exertion. She had an irregular tachycardia with no clinical sign of heart failure. The cardiothoracic ratio on chest $x$ ray had increased from $100 / 205 \mathrm{~cm}$ to $150 / 235$ $\mathrm{cm}$. The electrocardiogram showed an incessant arrhythmia in which most QRS complexes had a right bundle brunch block pattern (fig 8). Twenty four hour electrocardiographic recording showed frequent sustained arrhythmias of 260 beats per minute. There were never two consecutive sinus beats. The echocardiogram showed a dilated left ventricle with impaired contraction.

An invasive electrophysiological study confirmed fascicular ventricular tachycardia, each ventricular beat being preceded by a fascicular deflection but not by a His potential. Intravenous flecainide acetate abolished the arrhythmia and restored sinus rhythm. Oral flecainide maintained sinus rhythm with occasional ventricular bigeminy. Six weeks after the start of treatment the heart size returned to normal and echocardiography
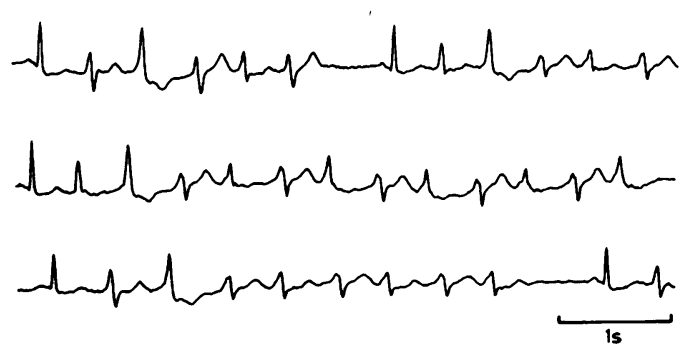

Figure 6 Ventricular tachycardia recorded during ambulatory electrocardiographic monitoring of the mother of the proband of family $B$. The ventricular tachycardia was non-sustained, at times bidirectional (middle trace), and at other times monomorphic (lower trace). 


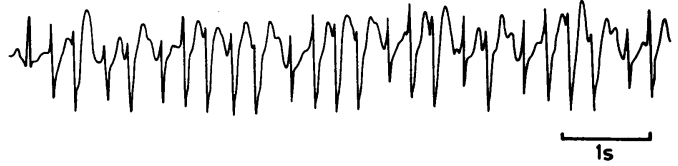

Figure 7 Ambulatory electrocardiographic recording from the proband of family $C$ showing an irregular, wide QRS tachycardia, confirmed at electrophysiological study to be ventricular tachycardia.

showed a considerable improvement in ventricular function. After two years the patient remains symptom free on flecainide. Her chest $x$ ray is normal and electrocardiographic monitoring shows sinus rhythm with frequent ventricular extrasystoles but without ventricular tachycardia.

The patient's mother first had palpitation 20 years ago and was treated with practolol for several years. At the time of investigation she was not taking medication. Her 24 hour electrocardiographic recording showed frequent, non-sustained, narrow $Q R S$ tachycardia occurring mainly at night. While this could be ventricular tachycardia, the precise nature of this arrhythmia has not been established because her symptoms do not warrant invasive investigation or treatment.

The mother's sister also gave a long history of palpitation. Her electrocardiogram recorded in 1970 showed non-sustained multiform ventricular tachycardia bearing a striking similarity to that of the proband. She had symptoms for 16 years despite treatment with quinidine. Recent reassessment showed frequent ventricular extrasystoles that continued after quinidine was stopped. Ventricular tachycardia was not present on 24 hour electrocardiographic monitoring. The resting electrocardiogram and cross sectional echocardiogram were normal. Treatment with flecainide initially reduced the ventricular ectopic activity but after some months her symptoms and the arrhythmia recurred. Other antiarrhythmic drug treatments are being investigated.

The maternal grandfather's family showed a strikingly high incidence of early death said to be due to heart disease but no further details are available (fig 1). The mother's cousins, both female, have also had arrhythmias. One cousin died suddenly at the age of 17 years and the other is alive and although she has symptoms she is not taking medication.

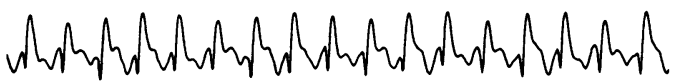

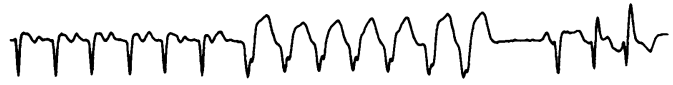

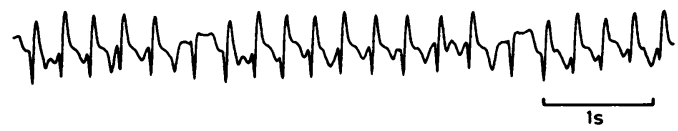

Figure 8 Ambulatory electrocardiographic recording from the proband of family $D$ showing a sustained, wide $Q R S$ tachycardia with varying $Q R S$ configuration, confirmed at electrophysiological study to be ventricular tachycardia.

\section{Discussion}

THE ARRHYTHMIAS AND THEIR PATHOPHYSIOLOGY

Understanding of the pathophysiology and electrophysiology of ventricular tachycardia in children has been hampered by its rarity. The long QT syndrome and torsade de pointes are now widely recognised but, although it is tempting to consolidate new cases with others previously described, the growing trend to soften the diagnostic criteria of the long QT syndrome may inappropriately amalgamate diverse pathophysiological conditions. It may be better to use the term "abnormal repolarisation syndromes" to cover both the classic long QT syndromes and patients with normal QT intervals but with abnormal $U$ waves, and to incorporate the possibility of abnormal repolarisation with a normal $\mathrm{QT}$ interval and normal U waves-a situation perhaps created by abnormally rapid repolarisation in parts of the heart, which produces pathological dispersion of repolarisation.

The cases of ventricular tachycardia presented are somewhat similar. All would satisfy even the most stringent definition of ventricular tachycardia. All occurred in young patients and in each there was evidence of a familial problem. Within each family there was a strikingly uniform type of arrhythmia. Yet, these interesting similarities are insufficient to establish a common electrophysiological or pathophysiological basis. The cases of ventricular tachycardia reported here are also recognisably different from the more common and apparently benign forms of repetitive monomorphic ventricular tachycardia seen in children without obvious heart disease.

Family A showed abnormal repolarisation with a normal QT interval but abnormal U waves. Since the original descriptions of the Jervell Lange-Nielsen ${ }^{1}$ and Romano Ward ${ }^{23}$ syndromes it has been apparent that other similar cases have variable inheritance or are sporadic ${ }^{4}$ and that an apparently similar clinical picture can be seen with a normal QT interval..$^{5-7}$ Some patients in the latter group, like those in family A, have large, prominent, and prolonged $\mathrm{U}$ waves. ${ }^{89} \mathrm{U}$ waves probably reflect repolarisation of the Purkinje system ${ }^{10}$ and abnormal $U$ waves may signify abnormal repolarisation, which is the probable underlying abnormality in the classic "long QT syndrome". "There is no consensus on when the $U$ wave is abnormal, either in height or duration. It has been suggested that $U$ waves can be regarded as normal when they are up to $50^{\circ}{ }^{\circ}$ of the height of the $\mathrm{T}$ wave and that a $\mathrm{U}$ wave "approaching the height of the T wave" should be considered abnormal and should then be included in measurement of the QT interval. ${ }^{10}$ The limits of normal QU duration are not known.

Family B also showed abnormal repolarisation. Though the multiform ventricular tachycardia and the abnormal $U$ wave might indicate an underlying abnormality of repolarisation, we were unable to show dispersion of ventricular refractoriness and there was no response to $\beta$ blockers. Multiform ventricular 
tachycardia and bidirectional ventricular tachycardia can coexist and both can also occur in the long QT syndrome. " Most of the few cases of bidirectional ventricular tachycardia reported in children have caused symptoms, with a high morbidity and mortality. ${ }^{11-13}$ Coumel et al have described a rare type of catecholamine induced ventricular tachycardia, induced by exercise or isoprenaline infusion, that progresses through bidirectional tachycardia to ventricular fibrillation. ${ }^{13}$

There is no direct evidence of abnormal repolarisation in families $C$ and $D$ but the incessant nature of the arrhythmias precluded such analysis. Though it may seem that the ventricular tachycardia in these two families might be a consequence of myocardial disease, the ventricular impairment was mild and may equally have been a result of the arrhythmia. ${ }^{14}$ Dilated cardiomyopathy may be familial and in some cases there is autosomal dominant inheritance. ${ }^{15}$ Familial cases are heterogeneous but they do show a strikingly high incidence of arrhythmias, syncope, and sudden death. ${ }^{16}$

Familial ventricular tachycardia has also been reported in association with arrhythmogenic right ventricular dysplasia when almost all those affected are male, ${ }^{17}$ in palmoplantar keratosis with right ventricular or biventricular disease,${ }^{19}$ in childhood periodic paralysis, ${ }^{20}$ and in various neuromuscular diseases with cardiac involvement. ${ }^{21}$

\section{GENETICS}

In each of these four families the proband is a female who presented in adolescence. Furthermore, it is apparent from fig 1 that there is a preponderance of affected females. Although the type of arrhythmia within each family is consistent, it is inappropriate to pool the families for analysis because there are clear distinctions between them. In particular, family $D$ is the only one in which there is obligate transmission of the gene from a male (I.6). In that male's sibship there were two early male deaths suggesting that in family $D$ this may well be a simple autosomal dominant and the preponderance of females in the observed generation is a chance finding.

In the other families the female predominance may be no more than a chance observation. There are relatively few males and there is no evidence of an excess number of miscarriages to suggest a more severe form of the disease causing early loss of affected males. The small number of families and the likelihood of heterogeneity prevent formal segregation analysis but the possibility of some form of matrilineal transmission deserves examination in future family studies.

There are several disorders that preferentially affect females or are transmitted in the female line. In congenital myotonic dystrophy, some form of maternofetal interaction is assumed because the disorder is invariably inherited from the mother. Maternofetal transmission of an infectious agent is possible; $\mathrm{Hb}_{3} \mathrm{Ag}$ positive hepatitis was described as a familial disorder transmitted in the female line before it was recognised that the virus was transferred at delivery. ${ }^{22}$ It has been suggested that familial ventricular tachycardia may result from familial transfer of neurotropic viruses causing selective damage of conducting tissue, but though this is theoretically possible there is no supportive evidence. ${ }^{23}$

Transmission in the female line is also characteristic of mitochondrial inheritance because mitochondria are derived almost exclusively from the ovum. There is now clear evidence that some cases of mitochondrial myopathy result from an abnormality in the mitochondrial genes, which are distinct from those of the nuclear genome. This mechanism of transmission is of potential relevance in view of the involvement of the conducting tissue in the Kearns-Sayre syndrome that has been shown to be associated with mitochondrial abnormality. ${ }^{24}$ These mechanisms would result in preferential transmission in the female line but not in an exclusive involvement of female offspring. A further observation of possible relevance is the suggestion that various cardiac malformations are associated with a much greater recurrence risk in the offspring of affected females than of affected males. ${ }^{25}$ No explanation for this excess is yet apparent.

Future studies of ventricular tachycardia should assess in further detail any relation between sex and the expression of transmission of a conduction defect. Clinicians should be aware of the need to inquire after, and where necessary to investigate, first degree relatives of individuals with ventricular tachycardia.

There is a clear need for more extensive family studies in ventricular tachycardia. The four families described in this report show that familial ventricular tachycardia is heterogeneous and that pooling of families is likely to be counterproductive, both to the delineation of the clinical phenotypes and to the possible localisation of the gene defects using DNA linkage studies.

We are grateful to Dr $S$ Hunter, Dr K Jennings, Dr D Krikler, Dr A Marshall, and Dr P G Rees for permission to report details of patients under their care and to Dr W D Alexander, Dr J E of patients under their care and to Dr W D Alexander, Dr J E for help in tracing the electrocardiograms of family members.

1 Jervell A, Lange-Nielsen F. Congenital deaf-mutism functional heart disease with prolongation of the Q-T functional heart disease with prolongation of the Q-

2 Romano C, Gemme G, Pongiglione R. Aritmie cardiache rare dell'eta pediatrica. II. Accessi syncopali per rare dell'eta pediatrica. II. Accessi syncopali per
fibrillazione ventriculare parossistica. Clin Pediatr 1963; 45:656-83.

3 Ward OC. New familial cardiac syndrome in children.J Irish Med Assoc 1964;54:103-6.

4 Schwartz PJ. Idiopathic long QT syndrome: progress and questions. Am Heart $J$ 1985;109:399-411.

5 Bricker JT, Garson A Jr, Gillette PC. A familial history of seizures associated with sudden cardiac deaths. Am J Dis Child 1984;138:866-8.

6 Rutter N, Southall DP. Cardiac arrhythmias misdiagnosed as epilepsy. Arch Dis Child 1985;60:54-70.

7 Schwartz PJ. The idiopathic long QT syndrome. Ann Intern Med 1983;99:561-2.

8 Shaw TRD. Recurrent ventricular fibrillation associated with normal QT intervals. $Q J$ Med 1981;200:451-62.

9 McRae JR, Wagner GS, Rogers MC, Cament RV. Paroxys mal familial ventricular fibrillation. J Pediatr 1974;84 515-8.

10 Garson A Jr. The electrocardiogram in infants and children: a systematic approach. Philadelphia: Lea and Febiger, systematic

11 von Bernuth G, Bernsau U, Gutheil H, et al. Tachyarrhythmic syncopes in children with structurally normal hearts
with and without QT-prolongation in the electrowith and without QT-prolongation in the

12 Gault JH, Cantwell J, Lev M, Braunwald E. Fatal familial 
cardiac arrhythmias. Histological observations on the cardiac conduction system. Am J Cardiol 1972;29:548-53.

13 Coumel P, Fidelle J, Lucet V, Attuel P, Bouvrain Y. Catecholamine-induced severe ventricular arrhythmias with Adams-Stokes syndrome in children: report of four cases. Br Heart J 1978;40(suppl):28-37.

14 Gallagher JJ. Tachycardia and cardiomyopathy: the chicken-egg dilemma revisited. J Am Coll Cardiol 1985; 6:1172-3.

15 Graber HL, Unverforth DV, Baker PB, Ryan JM, Baba N, Wooley CF. Evolution of a hereditary cardiac conduction and muscle disorder: a study involving a family with six and muscle disorder: a study involving a family

16 Kariv I, Kreister B, Sherf L, Feldman S, Rosenthal T. Familial cardiomyopathy. A review of 11 families. Am $j$ Familial cardiomyopathy.

17 Rakovec P, Rossi L, Fontaine G, Sasel B, Markez J, Voncina D. Familial arrhythmogenic right ventricular disease. $A m$ $J$ Cardiol 1986;58:377-8.

18 Ruder MA, Winston SA, Davis JC, Abbott JA, Eldar M Scheinman MM. Arrhythmogenic right ventricular dysplasia in a family. Am J Cardiol 1985;54:799-800.

19 Protonotarios N, Tsatsopoulou A, Patsourakos P, et al.
Cardiac abnormalities in familial pälmoplantar keratosıs. Br Heart J 1986;56:321-6.

20 Karpawich PP, Hart ZH, Perry BL, Chang C-H, Nigro MA, Green EW. Childhood periodic paralysis with dysrhythmias: electrophysiologic and

21 Dunnigan A, Benson DW Jr. Disturbances of cardiac rhythm. In: Pierpont MEM, Moller JH, eds. The genetics rhythm. In: Pierpont MEM, Moller JH, eds. The genetics of cardiovascular disease. Boston: Martinus Nijhoff,

22 Harper PS. Mendelian inheritance or transmissible agent?the lesson of Kuru and the Australia antigen. J Med Genet the lesson of Kur

23 James TN, MacLean WAH. Paroxysmal ventricular arrhythmias and familial sudden death associated with neural lesions in the heart. Chest 1980;78:24-30.

24 Egger J, Lake BD, Wilson J. Mitochondrial cytopathy. A multisystem disorder with ragged red fibres on muscle biopsy. Arch Dis Child 1981;56:741-52.

25 Nora JJ, Nora AH. Maternal transmission of congenital diseases: new recurrence risk figures and the question of cytoplasmic inheritance and vulnerability to teratogens. Am J Cardiol 1987;59:459-63. 